\title{
Reversible Data Hiding using QR Codes for Android
}

\author{
K.Lakshmi Sudha \\ Research scholor \\ Department of IT \\ SIES GST L, India
}

\author{
Kausthob Das \\ Department of IT \\ SIES GST L, India
}

\author{
Mahesh Notani \\ Department of IT \\ SIES GST L, India
}

\author{
Saisantosh \\ Shridhar \\ Department of IT \\ SIES GST L, India
}

\begin{abstract}
Initially barcodes have been widely used for unique product identification..QR (Quick response ) codes are 2D barcodes that can be used to embed not only product information, but also text, web URL, audio, video, phone contacts, and much more. Our project "Reversible data hiding using QR codes for android" is mainly concerned with generating and scanning of QR codes on android smart phones and also we planning to use QR code for authentication. The aim of our project is to compress the size of data thus helping the users to share data efficiently and make authentication more efficient using QR code. Our project aims at minimizing the memory requirements for storing different types of data on mobile phones, by directly redirecting to a specific webpage for the same.
\end{abstract}

\section{General Terms}

Reversible data hiding. QR Codes

\section{Keywords}

Reversible data hiding, Android smartphone, QR Codes, Authentication

\section{INTRODUCTION}

Barcodes have been used widely for the identification of products. In our project initially we plan to generate a QR code i.e. Quick Response code which is basically a twodimensional (2-D) barcode with added advantages over the traditional 1-D barcodes.We are developing an android application that would not only generate this QR code and but also be capable of scanning the generated QR codes as shown in Fig.1,successfully retrieving the information stored within the code.We also plan to include additional functionalities which would enable the use of this generated QR code for providing authentication to the users which helps in maintaining the confidentiality of the data. This application would be helpful in many ways and would have various advantages in our day to day life, as QR codes had been gaining massive popularity over years and have various useful applications.

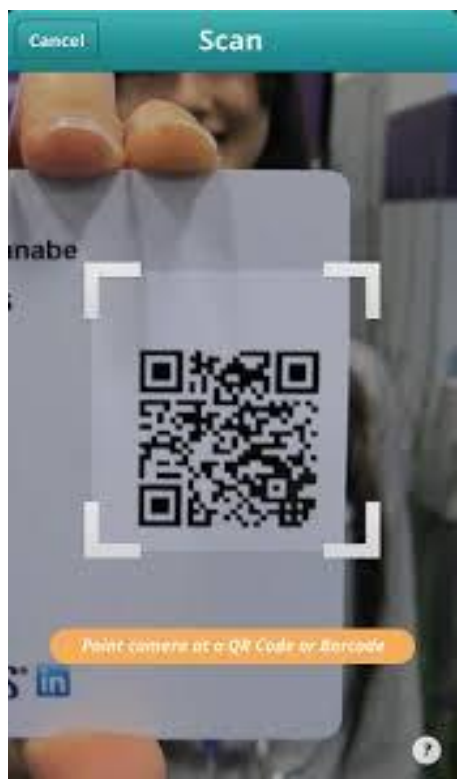

Fig 1: QR Code scanning Application

\section{RELATED WORK}

Cheh Huang,Feng-Cheng Chang,Wai-Chi Fang [1] explained the system Reversible data hiding with Histogram-Based Difference Expansion for QR Code Applications.But unfortunately the system Authentication support and QR code retrieval.

Nancy Victor [2] describes bit rate reduction to compress the size of data in the QR code and compress it to enhance the capacity of QR codes but the compression is lossy and the important data is lost.

Grillo A[3] tried increasing the space available for data, while preserving similar robustness, error correction and without losing compatibility with the original $\mathrm{QR}$ standard. The results show that $\mathrm{HCC} 2 \mathrm{D}$ leads to larger data density compared to $\mathrm{QR}$ at the price of a small computational overhead though the data density is slightly lower.

\section{COMPONENTS INVOLVED}

\subsection{Android Smartphone}

A smartphone is the basic requirement to run the android application.The ADK is installed such that the application will run on all android platforms. 


\subsection{Camera}

A camera in the android smartphone is the most necessary to scan the QR Code.The camera detects the QR code and it helps the application to run.

\section{GENERATION AND SCANNING OF QR CODE}

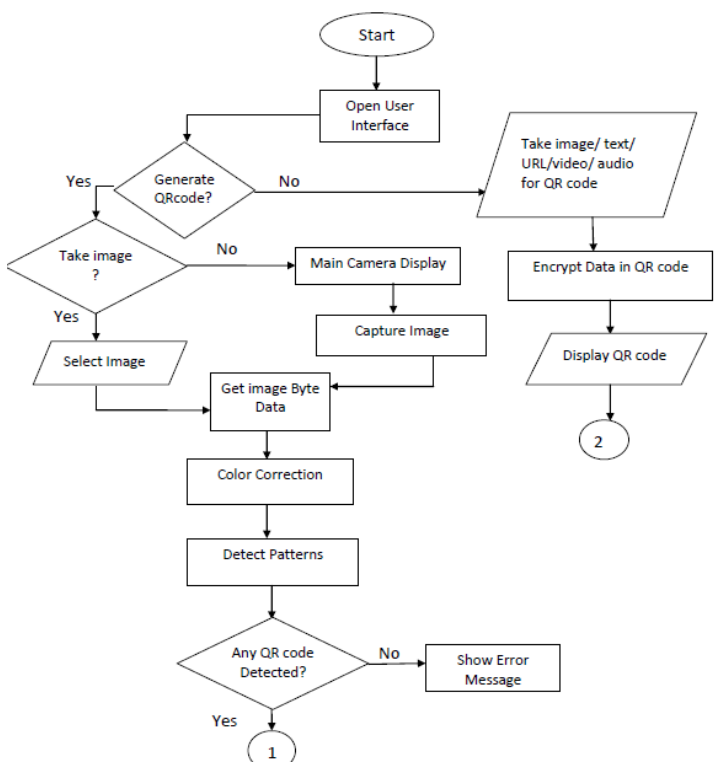

Fig 2: Flow chart for generating QR Code

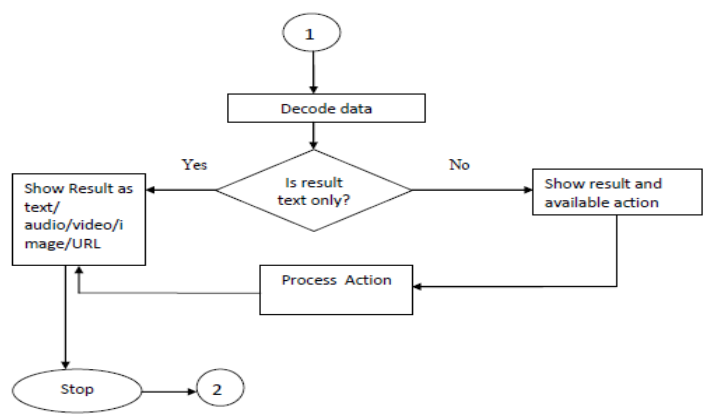

Fig 3. Flow chart for scanning QR Codes

\section{FEATUTURES PROVIDED}

\subsection{User Login}

$>\quad$ For every user the user will have to put the login id and password to access the application.

$>$ This ensures that only authorized users access the application and if the application is stolen or the phone is stolen the application cannot be misused.

\subsection{Authentication}

$>$ When we scan or generate QR code we are hiding the data in the QR code by encrypting it.

$>$ The algorithm used for encryption is AES.

$>$ A default key is used in AES and a 5 digit user key.
The default key and 5 digit key are used to encrypt the data and create another key which will be used for decryption.

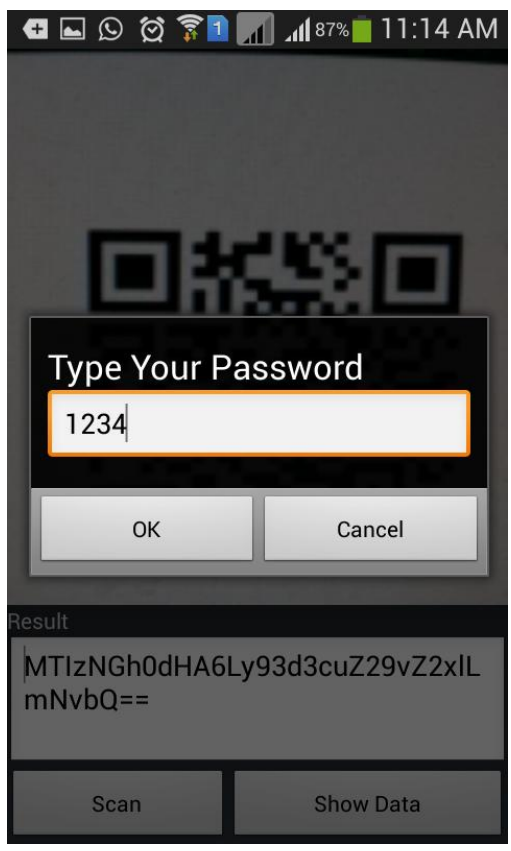

Fig 4: Authentication

$>$ The decryption uses that key and 5-digit user key to decrypt the data in the QR code.The default key size can be 128,192 or 256 bit.

\section{WORKING}

\subsection{IMPLEMENTATION OF SCANNING}

$>$ Start the Application in mobile,

$>$ Select "Scan QR Code"

$>$ Use the mobile camera to scan the QR Code.

$>$ Enter the 5-Digit key to access the data in QR Code.

$>$ Control the device by selection respective options from the menu as shown in Fig.2

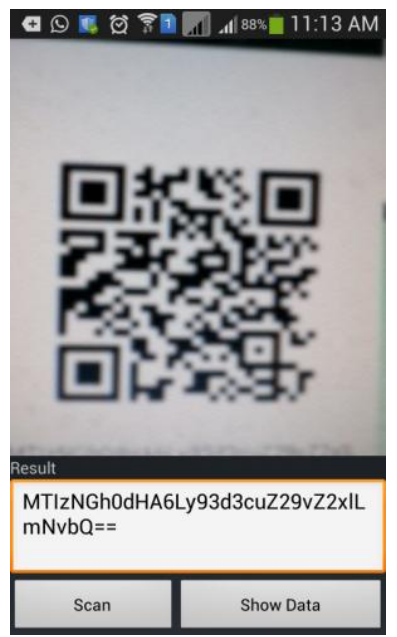

Fig 5:Scanning Implementation 


\subsection{IMPLEMENTATION OF GENERATION}

$>$ Start the android application.

$>$ Enter username and password.

$>$ Select "Generate QR Code"

$>$ Select the type of data to be encrypted.

Enter the data and select the color of QR Code.

$>$ Enter 5-digit password

$>$ Press Generate and the $\mathrm{QR}$ code is generated.

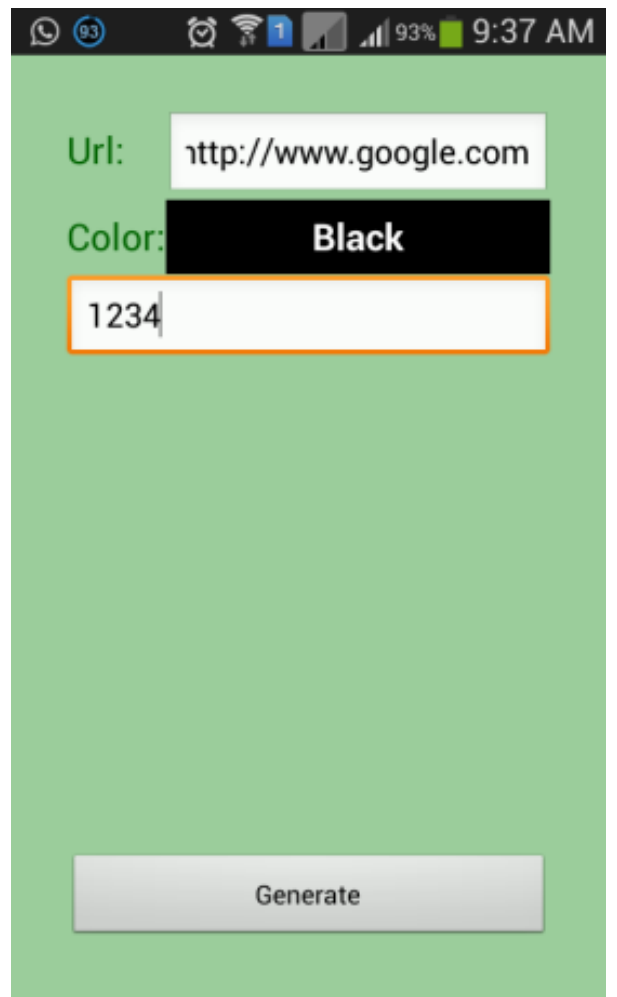

Fig 6:Generation Implementation

\section{RESULTS}

Reversible data hiding using QR codes for android is a human controlled android application that helps in hiding data within the QR code whether it is text,email,phone,sms and provides authentication to encrypt the data within the QR code. It is a system that was never developed previously with the main advantage of encryption to provide data security. Our results show that the application can scan and generate QR codes with encryption key and encrypt the data within it and decode it later using the same security key. The important aspect is that only the phone having the same application installed can decode the QR code.The implementation can create widespread impact in all aspects of human life making life a better place to live in.

\section{REFERENCES}

[1] Reversible data hiding with Histogram-Based Difference Expansion for QR Code Applications-Hsiang-Cheh Huang,Feng-Cheng Chang,Wai-Chi Fang,IEEE Transactions on Consumer Electonics,vol.57,No.2,May 2011.

[2] Enhancing the Data Capacity of QR Codes by Compressing the Data before Generation-Nancy Victor Centre for Information Technology and Engineering M.S. University

[3] High Capacity Colored Two Dimensional codes-Grillo, A. ; Dept. of Comput. Sci., Syst. \& Production, Univ. of "Tor Vergata", Rome, Italy ; Lentini, A. ; Querini, M. ; Italiano, G.F

[4] QR-code Generator, http://qrcode.kaywa.com/,2010.

[5] J. S. Pan, H. C. Huang, L. C. Jain, and W. C. Fang (editors), Intelligent Multimedia Data Hiding, Springer, Berlin-Heidelberg, Germany, Apr,2007.

[6] H. C. Huang and W. C. Fang, "Metadata-based image watermarking for copyright protection," Simulation Modelling Practice and Theory, vol.18, no. 4, pp. 436445, Apr. 2010

[7] J. S. Tan, "QR code," Synthesis Journal, Section 3, pp. 59-78, 2008.

[8] H. C. Huang and Y. H. Chen, "Genetic fingerprinting for copyright protection of multicast media," Soft Computing, vol. 13, no. 4, pp. 383-391, Feb. 2009.

[9] F. C. Chang, H. C. Huang, and H. M. Hang, "Layered access control schemes on watermarked scalable media," Journal of VLSI Signal Processing Systems for Signal, Image, and Video Technology, vol. 49,no. 3, pp. $443-$ 455, Dec. 2007.

[10] J. Tian, "Reversible data embedding using a difference expansion,'IEEE Trans. Circuits Syst. Video Technol., vol. 13 , no. 8, pp. 890-896,Aug. 2003.

[11] A. M. Alattar, "Reversible watermark using the difference expansion of a generalized integer transform," IEEE Trans. Image Process., vol. 13,no. 8, pp. 1147 1156, Aug. 2004.

[12] Z. Ni, Y. Q. Shi, N. Ansari, and W. Su, "Reversible data hiding," IEEE Trans. Circuits Syst. Video Technol., vol. 16, no. 3, pp. 354-362, March 2006

[13] D. M. Thodi and J. J. Rodriguez, "Expansion embedding techniques for reversible watermarking," IEEE Trans Image Process., vol. 16, no. 3, pp. 721-730, April 2007.

[14] H. J. Kim, V. Sachnev, Y. Q. Shi, J. Nam, and H. G. Choo, "A novel difference expansion transform for reversible data embedding," IEEETrans. Information Forensics and Security, vol. 3, no. 3, pp. 456-465,Sep. 2008 